\title{
Se necesita mejorar el proceso de elaboración de tesis en pregrado
}

\section{The undergraduate thesis preparation process needs to be improved}

La publicación de las tesis de pregrado en revistas científicas, es un asunto que merece reflexión. En primer lugar, revisar ¿cuál es la finalidad de la tesis?; en la mayoría de facultades de Medicina, la tesis es un trabajo de investigación, y se califican tanto el informe escrito y la presentación oral; de ello, podemos deducir que su finalidad es probar que el candidato puede hacer, y comunicar sus investigaciones.

Idealmente, todas las investigaciones deberían terminar en una publicación científica; sin embargo, no necesariamente todas las tesis son publicables. El editor de la revista Información Tecnológica, menciona en su editorial: "Las razones para esto son simples: los objetivos de una Tesis y los de una publicación no son necesariamente los mismos. Los factores de originalidad, aporte al conocimiento científico o tecnológico, y la documentación del trabajo no son siempre exigidos en un trabajo de Tesis de pregrado e incluso en algunas de magíster, pero son obligatorias en un artículo." (1), concepto que suscribo.

Varios factores pueden explicar la baja tasa de publicación, uno es que los formatos de presentación de tesis son distintos del formato del artículo científico que se publica en las revistas, exigiendo al egresado la adecuación del formato, para intentar su publicación (2). Es segundo factor, son los asesores; en algunas universidades, muchos de los asesores de tesis no han elaborado tesis ni publicado un artículo de investigación. Un estudio realizado por Pereyra y col (3), en docentes, encontró una asociación positiva entre haber publicado con ser menor de 40 años de edad, ser profesor en una universidad en la que se requiere una tesis para titularse y ser un profesor de una universidad productiva.

En este fascículo de la Revista Médica Herediana, se publica el artículo "Publicación de artículos científicos por asesores de tesis de una Facultad de Medicina" de Alarcon-Ruiz CA, y Quezada MA, cuyo objetivo fue determinar la frecuencia de publicación de artículos científicos por los asesores de tesis de pregrado de la carrera profesional de Medicina. La conclusión a la que llegan los autores es que la tasa de publicación en los últimos años de los asesores de tesis, es baja (4). Recomiendan, “...que las universidades tengan en cuenta al momento de la selección de docentes para asesorar una tesis, que posea conocimientos y experiencia en investigación no solo teóricos, sino también prácticos...que se vea reflejada en la productividad...y que...tengan publicaciones anuales" (4).

Disponer de investigadores activos para que asesoren tesis de pregrado es muy difícil, no sólo por el número de tesis anuales sino además en Medicina, por la diversidad de especialidades; esto es válido no sólo para Perú, sino también para otros países de Latinoamérica y el mundo.

Para obtener mejores resultados en las tasas de publicación de las tesis de pregrado, es necesario no sólo buenos asesores, sino mejorar la calidad de las tesis. Varias universidades y facultades de Medicina, tratando de mejorar la calidad, están permitiendo la elaboración de tesis en grupo de hasta tres o cuatro estudiantes y asignan dos asesores: uno en metodología y otro de la especialidad tema de investigación.

Es necesario recordar que, la elaboración y sustentación de la tesis, es un proceso o subproceso ubicado hacia el final del macroproceso de formación profesional, requerido para la obtención del título o licenciatura, por ello, para mejorar la calidad, se requiere de un enfoque de gestión por procesos, es decir, establecer una secuencia de actividades que conduzcan a la elaboración, ejecución del proyecto, elaboración del informe y sustentación de la tesis. Además, establecer objetivos, indicadores y metas claros. 
Si el objetivo de la Facultad y Universidad es que las tesis se publiquen en revistas científicas, tienen que mejorar el proceso de elaboración y sustentación de la tesis; es necesario fomentar la investigación relevante, el trabajo en equipo (permitiendo tesis en grupos de estudiantes), mejorar la calidad de la investigación, aumentar las capacidades de los miembros de los jurados o evaluadores de las tesis y modificar la estructura y el formato de presentación de la tesis, por mencionar los más importantes.

"Si los resultados de una tesis no se publican, no pueden ser considerados como validados. A lo más servirán para que el estudiante cumpla la etapa final de sus estudios y reciba su anhelado título o grado" (1).

La Ley Universitaria peruana, obliga a las universidades a publicar y mantener las tesis en un repositorio institucional, lo que ya es un gran avance para la visibilidad de las tesis. Para que la tesis pueda ser publicada en una revista científica es necesario un paso más, adecuar el formato al de un artículo científico, tal como se mencionó anteriormente. Hay que tener en cuenta que, a diferencia de un artículo científico, una tesis puede abordar más de un tema y presentar más de un enfoque de algunos temas, y suelen ser más extensos, lo que hace más difícil la tarea. Algunas facultades ya han cambiado el formato de presentación de la tesis de pregrado al de artículo científico, lo que aumenta la probabilidad de ser presentado a una revista para su publicación.

Juan Miyahira ${ }^{1}$

\section{REFERENCIAS BIBLIOGRÁFICAS:}

1. Las Tesis No Son Todas Publicables. Inf tecnol. 2010; 21(6):1-1. doi: http://dx.doi.org/10.4067/ S0718-07642010000600001.

2. Miyahira J. ¿Por qué la tasa de publicación de las tesis en Medicina es baja? Posibles explicaciones. Rev Med Hered. 2015; 26:207-208.
3. Pereyra-Elias R, Huaccho-Rojas JJ, Taype-Rondan A, Mejia CR, Mayta-Tristán P. Publicación y factores asociados en docentes universitarios de investigación científica de escuelas de medicina del Perú. Rev perú med exp salud publica. 2014; 31(3):424-430.

4. Alarcon-Ruiz CA, Quezada MA. Publicación de artículos científicos por asesores de tesis de una Facultad de Medicina. Rev Med Hered. 2018; 29:152157.

1 Editor Jefe. Revista Médica Herediana. 